\title{
Long-term clinical outcome of patients with severe atherosclerotic renal artery stenosis after percutaneous transluminal renal angioplasty
}

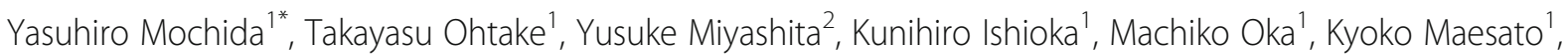
Hidekazu Moriya ${ }^{1}$, Sumi Hidaka', Shigeru Saito ${ }^{3}$ and Shuzo Kobayashi ${ }^{1}$

\begin{abstract}
Background: In the patients with severe atherosclerotic renal artery stenosis (ARAS), the evaluation of long-term clinical outcome after percutaneous transluminal renal angioplasty (PTRA) has yet been insufficient. The aim of this study was to focus on only patients with severe ARAS and to evaluate the more long-term clinical outcome after PTRA.

Methods: We performed a single-center retrospective review of the outcome after PTRA. All eligible patients had ARAS of $\geq 75 \%$ by catheter renal angiography and underwent PTRA in our hospital from July 2004 to December 2007. We investigated long-term changes of renal function and the other clinical outcomes in patients with severe ARAS.

Results: The median follow-up period was 83 months. The overall mean stenosis rate was $87 \%$. There were 24 patients with $75 \leq$ and $<90 \%, 38$ patients with $90 \leq$ and $<95 \%$, and 12 patients with $\geq 95 \%$ stenosis. Serum creatinine(S-Cr) levels were from $1.27 \pm 0.68$ at baseline to $1.33 \pm 0.65 \mathrm{mg} / \mathrm{dl}$ at 84 months after PTRA in overall $(p=0.255)$. The renal size was also preserved for long-term in overall. Although serum creatinine level in patients with renal major axis $<85 \mathrm{~mm}$ at PTRA significantly increased $(p<0.05)$ after 60 months post PTRA, it did not worsen in patients with renal major axis more than $85 \mathrm{~mm}$ during observational period. The number of anti-hypertension drugs decreased from baseline $(p=0$. 0037), and both systolic and diastolic blood pressure significantly decreased $(p<0.001)$ after PTRA. Finally, 4 patients had renal death, and 14 patients died. Overall survival for 60 months was 85\%.
\end{abstract}

Conclusions: We demonstrated that PTRA for patients with severe ARAS significantly decreased blood pressure, and preserved renal function and renal size for long term.

Trial registration: This study was admitted by Tokushukai Group Institutional Review Board. (IRB no. TGE00532-024)

Keywords: Atherosclerotic renal artery stenosis (ARAS), Percutaneous transluminal renal angioplasty (PTRA), Renal outcome, Mortality, Atherosclerosis

\footnotetext{
* Correspondence: yasuhiro.mochida@tokushukai.jp

${ }^{1}$ Kidney Disease and Transplant center, Shonan Kamakura General Hospital,

1370-1 Okamoto, Kamakura, Kanagawa 247-8533, Japan

Full list of author information is available at the end of the article
} 


\section{Background}

Some studies [1-4] have shown that PTRA improves renal function in patients with renal artery stenosis. However, current treatment of atherosclerotic renal artery stenosis (ARAS) has shifted towards medical therapy. In patients with ARAS, PTRA was concluded to be not superior to medical therapy for preserving renal function and improving the renal death rate and cardiovascular event rate based on the results of two multicenter randomized intervention trials (The Angioplasty and Stenting for Renal Artery Lesions (ASTRAL) study [5] and The Cardiovascular Outcomes in Renal Atherosclerotic Lesions (CORAL) study [6]). However, almost $40 \%$ of the renal arteries treated by PTRA had only mild stenosis $(<70 \%)$ in the ASTRAL study. In addition, the severity of RAS in some patients was evaluated by only Doppler echo or MRA (no angiographic evaluation). Furthermore, only $10 \%$ of the patients were followed for 60 months in the CORAL study. The median periods and median stenosis rate were 33.6 months, $75 \%$ and 43 months, $67 \%$ in ASTRAL and CORAL study, respectively.

Considering such problems with the preceding multicenter randomized trials, it should not necessarily be concluded that PTRA is not superior to medical therapy for ARAS for long-term. In a recent prospective interventional study (Japan multi-central Renal Artery Stent study) [7], the renal outcome and blood pressure control were reported to be good in ARAS patients with severe stenosis who underwent PTRA. However, the observation period of this study was only 1 year.

Our study evaluated the long-term clinical outcome only in patients with severe ARAS who underwent PTRA. The aim of this study was to focus on only patients with severe ARAS and evaluate the more longterm clinical outcomes after PTRA.

\section{Methods}

\section{Study design and patients}

This was a single-center retrospective cohort study of patients who underwent PTRA (balloon dilation and stent placement) at Shonan Kamakura General Hospital from July 2004 to December 2007. Patients were followed up to the end of April 2015 and collected information about serum creatinine, renal death, heart failure, cardiac death, and all-cause death from the electronic medical records. This study was approved by local ethical committee (IRB no. TGE00532-024).

Patients with clinical findings such as uncontrolled refractory hypertension, renal dysfunction, and heart failure were screened by Doppler ultrasound and/or magnetic resonance imaging of the renal artery to detect ARAS. Patients with angina pectoris or myocardial infarction were also screened by using the same methods in consideration of the high prevalence of systemic atherosclerotic disease. At the time of coronary angiography, renal angiography was concomitantly done in patients who had been suspected to have ARAS. The diagnostic angiography and therapeutic catheter intervention were done separately. Quantitative computerassisted angiography (on-line quantitative coronary analysis system on Philips H5000: Philips Co., Ltd., Tokyo, Japan) was used to measure the degree of stenosis and was evaluated by two independent cardiologists to reduce mistake of evaluating the extent of renal stenosis. Based on the renal angiographic findings, PTRA was indicated for renal artery stenosis $\geq 75 \%$. PALMAZ ${ }^{\circ}$ stent and renal protect devices were used in all patients. In patients with bilateral ARAS with $\geq 75 \%$ stenosis, PTRA was done for both stenotic arteries, separately. Patients with technical failure (defined as $>25 \%$ residual stenosis on final angiography after intervention) and patients who were lost to follow-up within 12 months were excluded from analysis. This study included the patients who died or showed complications or renal death within 12 months after PTRA.

\section{Data collection}

Baseline demographic characteristics, serum creatinine, the major axis of the kidney on ultrasound or CT, the peak systolic velocity (PSV), end-diastolic velocity and resistive index (RI) on ultrasound, and the extent of RAS on catheter angiography before PTRA were recorded. In patients with bilateral RAS, stenosis grouping was performed on the basis of the more severe stenosis. Patients were reviewed at 3 and 6 months after PTRA and then every 12 months. Ambulatory blood pressure and serum creatinine were measured at every visit in all patients. Doppler echography was performed every 6 months after PTRA. Renal angiography was done in patients with high PSV of renal artery $>180 \mathrm{~cm} / \mathrm{s}$ or the ratio of renal PSV to aortic PSV (the renal-aortic ratio; RAR, i.e., the ratio of the PSV in the renal artery to the PSV in the aorta) $>3.5$.

\section{Definitions}

Severe RAS was defined as the stenotic rate on catheter angiography more than $75 \%$. Patients with severe stenosis ( $\geq 75 \%$ stenosis) was divided into three categories, i.e., lowgrade group ( $75 \leq$ and $<90 \%$ stenosis), intermediate-grade group ( $90 \leq$ and $<95 \%$ stenosis), and high-grade group ( $\geq$ $95 \%$ stenosis). Technical success was defined as $\leqq 25 \%$ residual RAS at final angiography, while all other outcomes were defined as technical failure. Restenosis was defined as recurrence of $\geq 75 \%$ luminal restenosis on renal angiography. Renal death was defined as an estimated glomerular filtration rate $(\mathrm{eGFR}) \leqq 15 \mathrm{~mL} / \mathrm{min} / 1.73 \mathrm{~m}^{2}$ or initiation of dialysis. Uncontrolled refractory hypertension was defined as uncontrolled hypertension (>140/90 $\mathrm{mmHg}$ ) even if patients have three kinds of anti-hypertension drugs. 


\section{Study end-points}

The primary end-point was the change of serum creatinine after PTRA. Serum creatinine was evaluated at every 12 months and was compared with the baseline value in each patient. Secondary end-points included the changes of renal major axis (renal size), blood pressure, left ventricular (LV) mass on echocardiography, and outcomes including renal death, heart failure, cardiovascular death, and all-cause mortality. Interventional complications and restenosis after PTRA were also assessed.

It remains unresolved which renal size is required to preserve renal function after PTRA from the standpoint of long-term renal outcome. Therefore, for assessing the influence of renal size on long-term renal outcome, renal size before PTRA was divided into three categories $(85 \mathrm{~mm}<, 85 \leq<100 \mathrm{~mm}, 100 \mathrm{~mm} \leq)$ in this study.

\section{Statistical analysis}

Results are expressed as the mean \pm standard deviation (SD). Patient characteristics were compared using one-way analysis of variance (ANOVA). Continuous variables were compared using Wilcoxon signed-rank test or repeated ANOVA. After probability, $p$ value was less than 0.05 by ANOVA, the Bonferroni test was used for post hoc analysis. Kaplan-Meier curves were constructed for all-cause mortality. $P$ values $<0.05$ were considered to indicate significance. All statistical analyses were done with IBM SPSS Statistics Software Ver.21 (SPSS Inc. Chicago, Il).

\section{Results}

\section{Patients}

Based on the indication criteria for PTRA, 80 ARAS patients underwent PTRA at Shonan Kamakura General Hospital during a period of 42 months between July 2004 and December 2007. Diagnosis of ARAS was made at the admission to evaluate atherosclerotic coronary or peripheral arterial stenosis in 60 patients $(75 \%, 60 / 80$ patients), renal dysfunction without proteinuria and/or hematuria in 14 patients $(17.5 \%, 14 / 80)$, uncontrolled hypertension in 5 patients $(6.3 \%, 5 / 80)$, and congestive heart failure in 1 patient $(1.2 \%, 1 / 80)$. All these 80 patients underwent renal angiography for final diagnosis of ARAS. Six patients were excluded from analysis, because one patient had PTRA failure and five patients had a short follow-up period of $<12$ months. Finally, the remaining 74 patients were analyzed (Fig. 1). Three patients among them showed complications within 12 months.

\section{Demographic and other characteristics}

The characteristics of the 74 patients are shown in Table 1. Their mean age was $72 \pm 7$ years. Among them, $75 \%$ (55/74 patients) had a history of smoking, 34\% (25 patients) had diabetes mellitus, 92\% (68 patients) had a history of ischemic heart disease, and 19\% (14 patients) had a history of cerebrovascular disease. In addition, 55 patients (76\%) had unilateral ARAS and 18 patients $(24 \%)$ had bilateral ARAS. The median major renal axis was $94 \mathrm{~mm}$, while the mean peak systolic velocity, end-diastolic velocity, and resistive index (RI) of the renal artery were $254 \pm 99 \mathrm{~cm} / \mathrm{s}, 65 \pm 9 \mathrm{~cm} / \mathrm{s}$, and $0.75 \pm 0.10$, respectively.

There were 24 patients with $75 \leq$ and $<90 \%$ stenosis (low-grade group), 38 patients with $90 \leq$ and $<95 \%$ stenosis (intermediate-grade group), and 12 patients with $\geq$ 95\% stenosis (high-grade group). The overall mean stenosis rate was $87 \pm 8.7 \%$. The median observation period was 83 months (IQR 49,106).

\section{Primary end-point \\ Serum creatinine}

Serum creatinine levels were preserved over 84 months in overall and in each group with unilateral or bilateral

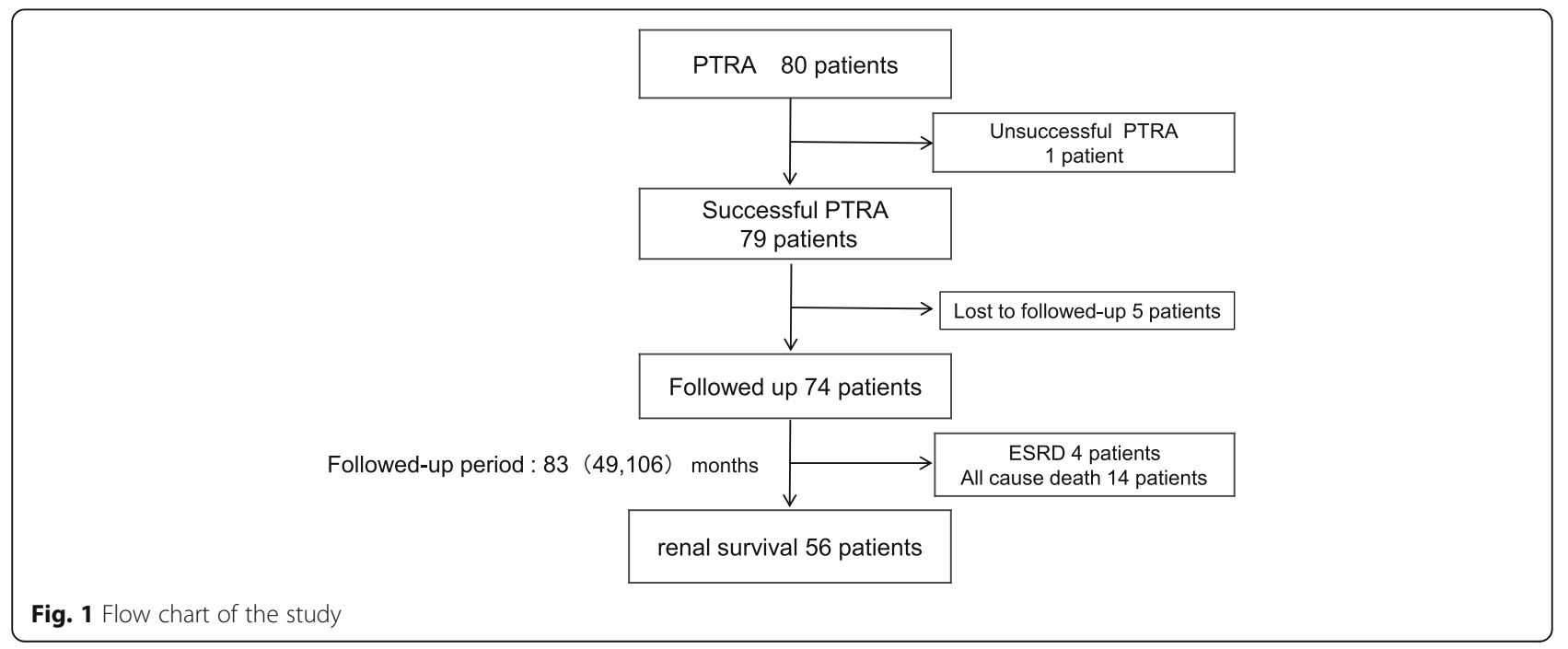


Table 1 Baseline characteristics

\begin{tabular}{|c|c|c|c|c|c|}
\hline \multirow{4}{*}{ Baseline Characteristics } & \multicolumn{5}{|c|}{ Stenosis grade } \\
\hline & \multirow{3}{*}{$\begin{array}{l}\text { All } \\
N=74\end{array}$} & \multirow{3}{*}{$\begin{array}{l}\text { Low } \\
75 \leq,<90 \% \\
N=24(33 \%)\end{array}$} & \multirow{3}{*}{$\begin{array}{l}\text { Intermediate } \\
90 \leq,<95 \% \\
N=38(51 \%)\end{array}$} & \multirow{3}{*}{$\begin{array}{l}\text { High } \\
95 \% \leq \\
N=12(16 \%)\end{array}$} & \multirow{3}{*}{$p$ value } \\
\hline & & & & & \\
\hline & & & & & \\
\hline Stenosis rate (\%) & $87 \pm 8.7$ & & & & \\
\hline Age (year) & $72 \pm 7$ & $72 \pm 5$ & $72 \pm 7$ & $72 \pm 8$ & 0.95 \\
\hline Male (\%) & 70 & 75 & 68 & 83 & 0.59 \\
\hline Smoking (\%) & 75 & 65 & 54 & 67 & 0.73 \\
\hline \multicolumn{6}{|l|}{ Comorbidities (\%) } \\
\hline DM & 34 & 29 & 32 & 50 & 0.43 \\
\hline Hypertension & 100 & 100 & 100 & 100 & - \\
\hline Hyperlipidemia & 61 & 63 & 56 & 75 & 0.48 \\
\hline IHD & 92 & 92 & $97^{*}$ & $75^{* *}$ & $0.047^{\dagger}$ \\
\hline CVD & 19 & 17 & 16 & 33 & 0.38 \\
\hline PAD & 36 & 42 & 34 & 33 & 0.82 \\
\hline \multicolumn{6}{|l|}{ Blood pressure } \\
\hline Systolic (mmHg) & $147 \pm 22$ & $145 \pm 21$ & $145 \pm 20$ & $160 \pm 27$ & 0.10 \\
\hline Diastolic (mmHg) & $79 \pm 12$ & $79 \pm 14$ & $79 \pm 9$ & $84 \pm 17$ & 0.45 \\
\hline \multicolumn{6}{|l|}{ Laboratory data } \\
\hline BUN (mg/dl) & $22.5 \pm 11.7$ & $19.2 \pm 7.9$ & $21.6 \pm 11.8$ & $31.0 \pm 16.7$ & 0.07 \\
\hline Creatinine (mg/dl) & $1.27 \pm 0.68$ & $1.20 \pm 0.59^{*}$ & $1.10 \pm 0.35^{*}$ & $1.95 \pm 1.14^{* *}$ & $0.0004^{\dagger}$ \\
\hline eGFR (mL/min/1.73 m2) & $47.9 \pm 16.1$ & $49.3 \pm 15.0$ & $51.1 \pm 14.5$ & $35.2 \pm 18.2$ & $0.0087^{\dagger}$ \\
\hline Albumin (g/dl) & $4.0 \pm 0.4$ & $4.0 \pm 0.4$ & $4.1 \pm 0.4$ & $3.8 \pm 0.7$ & 0.43 \\
\hline LDL-Cho (mg/dl) & $99 \pm 27$ & $90 \pm 25^{*}$ & $98 \pm 23$ & $118 \pm 36^{* *}$ & $0.017^{\dagger}$ \\
\hline U-TP (g/gcr) & $1.5 \pm 0.3$ & $0.29 \pm 0.74^{*}$ & $0.22 \pm 0.62^{*}$ & $1.21 \pm 1.44^{* *}$ & $0.002^{\dagger}$ \\
\hline \multicolumn{6}{|l|}{ Stenosis findings } \\
\hline Lateral RAS (\%) & 76 & $87^{*}$ & 76 & $50^{* *}$ & $0.047^{\dagger}$ \\
\hline Bilateral RAS (\%) & 24 & $13^{*}$ & 24 & $50^{* *}$ & \\
\hline Renal size $(\mathrm{cm})$ & $9.4 \pm 0.9$ & $9.5 \pm 0.8$ & $9.2 \pm 1.0$ & $8.8 \pm 0.7$ & 0.10 \\
\hline PSV $(\mathrm{cm} / \mathrm{s})$ & $254 \pm 99$ & $233 \pm 54^{*}$ & $280 \pm 106^{*}$ & $199 \pm 120^{* *}$ & $0.032^{\dagger}$ \\
\hline $\operatorname{EDV}(\mathrm{cm} / \mathrm{s})$ & $65 \pm 9$ & $57 \pm 11^{*}$ & $77 \pm 15^{*}$ & $40 \pm 22^{* *}$ & $0.011^{\dagger}$ \\
\hline Resistive index & $0.75 \pm 0.10$ & $0.75 \pm 0.09$ & $0.73 \pm 0.08$ & $0.79 \pm 0.08$ & 0.14 \\
\hline \multicolumn{6}{|l|}{ Medical therapy (\%) } \\
\hline ARB & 45 & 42 & 47 & 33 & 0.58 \\
\hline ACEi & 27 & 42 & 16 & 33 & 0.10 \\
\hline Ca blocker & 65 & 58 & 63 & 83 & 0.48 \\
\hline$\beta$ or $\alpha / \beta$ blocker & 38 & 46 & 32 & 42 & 0.76 \\
\hline Statin & 58 & 50 & 61 & 67 & 0.13 \\
\hline \multicolumn{6}{|l|}{ Observational period } \\
\hline Median (IQR), months & $83(49,106)$ & $89(51,114)$ & $76(48,105)$ & $80(56,95)$ & 0.69 \\
\hline
\end{tabular}

Data were expressed as mean \pm SD or \%

Expanded abbreviation; RAS renal artery stenosis, DM diabetes meliitus, IHD ischemic heart disease, CVD cerebral vascular disease, $P A D$ peripheral arterial disease, eGFR estimated glomerular filtration rate, U-TP urine total protein, PSV peak systolic velocity, EDV end-diastolic velocity, ARB angiotensin type II receptor blocker, ACEi angiotensin converting enzyme inhibitor

${ }^{\dagger} P$ value was less than 0.05 by one-way analysis of variance

$P$ value between ${ }^{* *}$ and * was less than 0.05 with post-hoc the Bonferroni test 
ARAS (Fig. 2). Serum creatinine levels from baseline to the final hospital visit ranged from $1.27 \pm 0.68$ to $1.33 \pm$ $0.65 \mathrm{mg} / \mathrm{dl}$ in the overall, $1.21 \pm 0.59$ to $1.47 \pm 0.91 \mathrm{mg} /$ $\mathrm{dl}$ in the low-grade group, $1.10 \pm 0.34$ to $1.89 \pm 1.84 \mathrm{mg} /$ $\mathrm{dl}$ in the intermediate-grade group, and $1.95 \pm 1.14$ to $1.76 \pm 0.80 \mathrm{mg} / \mathrm{dl}$ in the high-grade group. In patients with unilateral ARAS, serum creatinine changed $1.21 \pm$ 0.63 to $1.28 \pm 0.66 \mathrm{mg} / \mathrm{dl}$ in the low-grade group, $1.03 \pm$ 0.29 to $1.16 \pm 0.91 \mathrm{mg} / \mathrm{dl}$ in the intermediate-grade group, and $2.43 \pm 1.44$ to $1.64 \pm 0.64 \mathrm{mg} / \mathrm{dl}$ in the high-grade group, while in patients with bilateral ARAS, serum creatinine changed $1.20 \pm 0.32$ to $1.12 \pm 0.41 \mathrm{mg} / \mathrm{dl}$ in the low-grade group, $1.34 \pm 0.45$ to $1.52 \pm 0.31 \mathrm{mg} / \mathrm{dl}$ in the intermediate-grade group, and $1.46 \pm 0.47$ to $0.76 \mathrm{mg} / \mathrm{dl}$ in the high-grade group. In eight patients with a serum creatinine level $>1.2 \mathrm{mg} / \mathrm{dl}$ in the high-grade group, creatinine showed a significant decrease from $2.45 \pm 1.07 \mathrm{mg} / \mathrm{dl}$ to $1.76 \pm 0.64 \mathrm{mg} / \mathrm{dl}$ at 12 months after PTRA $(p=0.017)$ and was maintained for 24 months after PTRA (1.74 \pm $0.53 \mathrm{mg} / \mathrm{dl}, p=0.025$ ) (Additional file 1: Figure S1).

\section{Secondary end-points}

\section{Renal size (renal major axis)}

Renal major axis was $94 \pm 9 \mathrm{~mm}$ at baseline, $98 \pm 9 \mathrm{~mm}$ after 36 months, and $96 \pm 9 \mathrm{~mm}$ after 60 months, respectively. Renal size was not significantly different between baseline and 60 months after PTRA $(p=0.133)$.
The renal size significantly enlarged at 36 months after PTRA compared with baseline $(p<0.01)$ (Additional file 2: Figure S2).

Next, we divided renal size into three categories and evaluated the influence of renal size at PTRA on longterm renal function. Three categories of renal size and the number of patients were $85 \mathrm{~mm}<(N=11), 85 \leq<$ $100 \mathrm{~mm}(N=38)$, and $100 \mathrm{~mm} \leq(N=14)$. As a result, serum creatinine levels significantly increased $(p<0.05)$ in patients with renal major axis $<85 \mathrm{~mm}$ after 60 months post PTRA. However, the levels of serum creatinine did not change throughout 84 months in the other groups (renal major axis with $85 \mathrm{~mm}$ or more) (Fig. 3).

\section{Blood pressure}

At baseline, the mean systolic blood pressure was $147 \pm$ $22 \mathrm{mmHg}$ and the mean diastolic blood pressure was 79 $\pm 12 \mathrm{mmHg}$. Both systolic and diastolic blood pressure significantly decreased at 12 and 60 months after PTRA compared with baseline $(p<0.001)$ (Fig. 4$)$. At 12 months after PTRA, the number of anti-hypertension drugs significantly decreased from baseline (from $1.95 \pm 0.8$ to $1.70 \pm 1.0$ drugs, $p<0.01$ ).

\section{Heart failure and LV mass}

During the observation period, admission for heart failure was required in 17 patients $(24 \%)$ and all of them

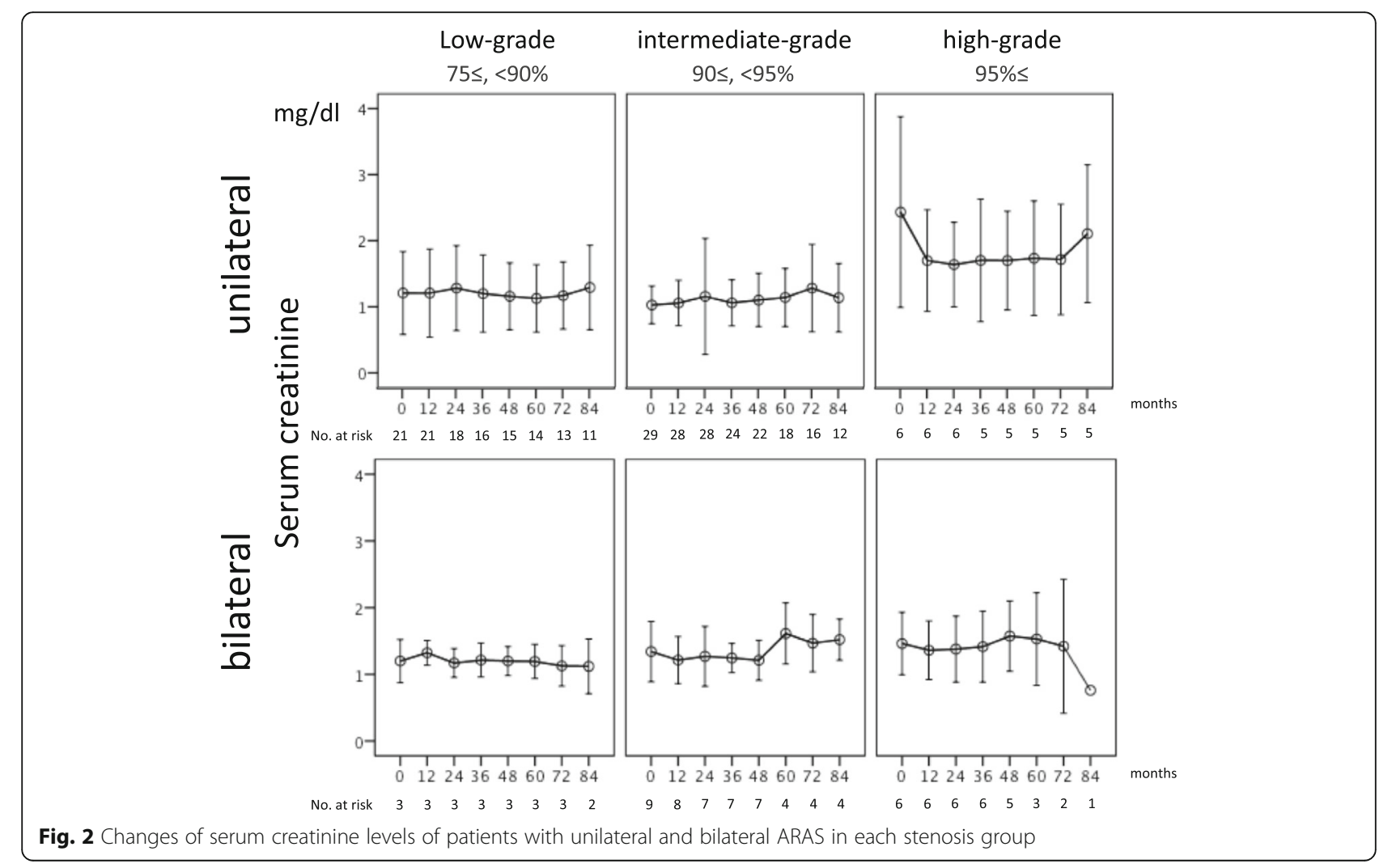




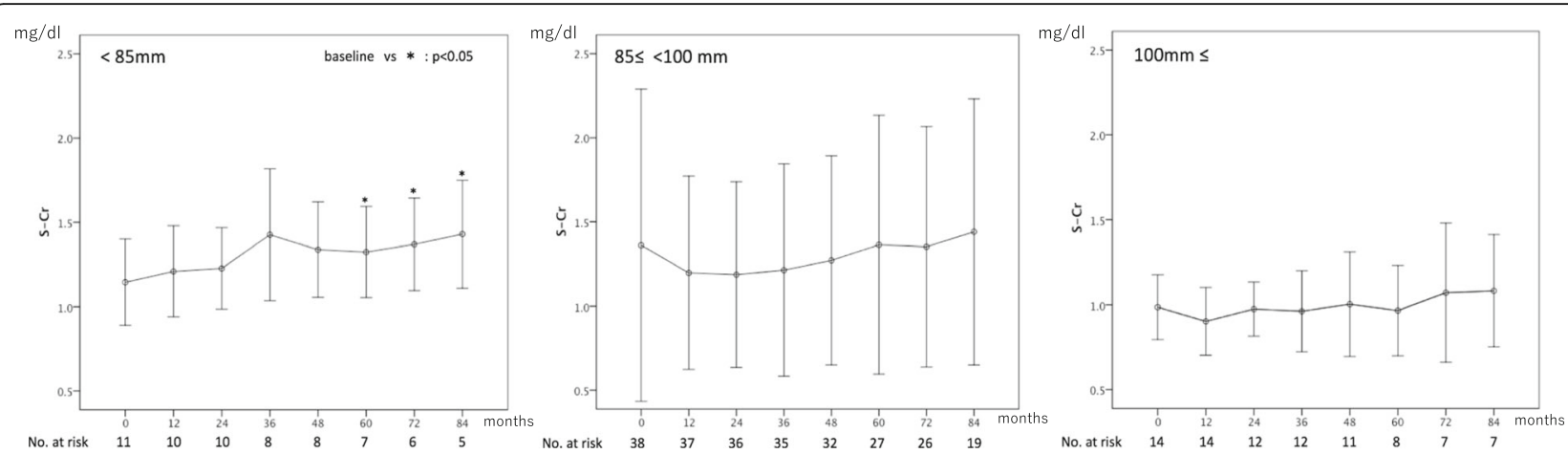

Fig. 3 Change of serum creatinine in three different renal size groups. $※ p<0.05$ vs. the pretreatment level

were admitted to our hospital. Among the 74 patients, $60 \%$ showed regression of LV mass at 12 months after PTRA compared with baseline. However, there was no significant difference between mean baseline LV mass and that at 12 months after PTRA (baseline: $172 \pm 43 \mathrm{~g}$, 12 months: $168 \pm 53 \mathrm{~g}, p=0.90$ ). Changes of LV mass from baseline to 12 months after PTRA were significantly associated with the changes of both systolic and diastolic blood pressure from baseline to 12 months after PTRA (systolic; $r=0.403, p=0.002$, diastolic; $r=$ $0.284, p=0.04)$.

\section{Renal death (need renal replacement therapy)}

During the observational period, renal death occurred in $5 \%$ (four patients). All patients with renal death initiated hemodialysis and survived until the end of the observation period. The cause of renal death was cholesterol crystal embolism (CCE) in two patients (for detail, see CCE in complications) and progressive renal failure due to ischemic nephropathy in two patients.

\section{All-cause mortality}

At the end of observational period, all-cause mortality rate was $19 \%$ (14 patients). The cause of death was cardiovascular disease in 6 patients $(43 \%)$, cancer in 5 patients (36\%), infection in 2 patients (14\%), and mesenteric artery infarction due to CCE in 1 patient (2\%) (Table 2). Overall survival rate for 60 months was $85 \%$.

\section{Complications}

CCE

CCE occurred in 4 out of 74 patients (5.4\%) (Table 2), with renal CCE (2 patients) and mesenteric necrosis (1
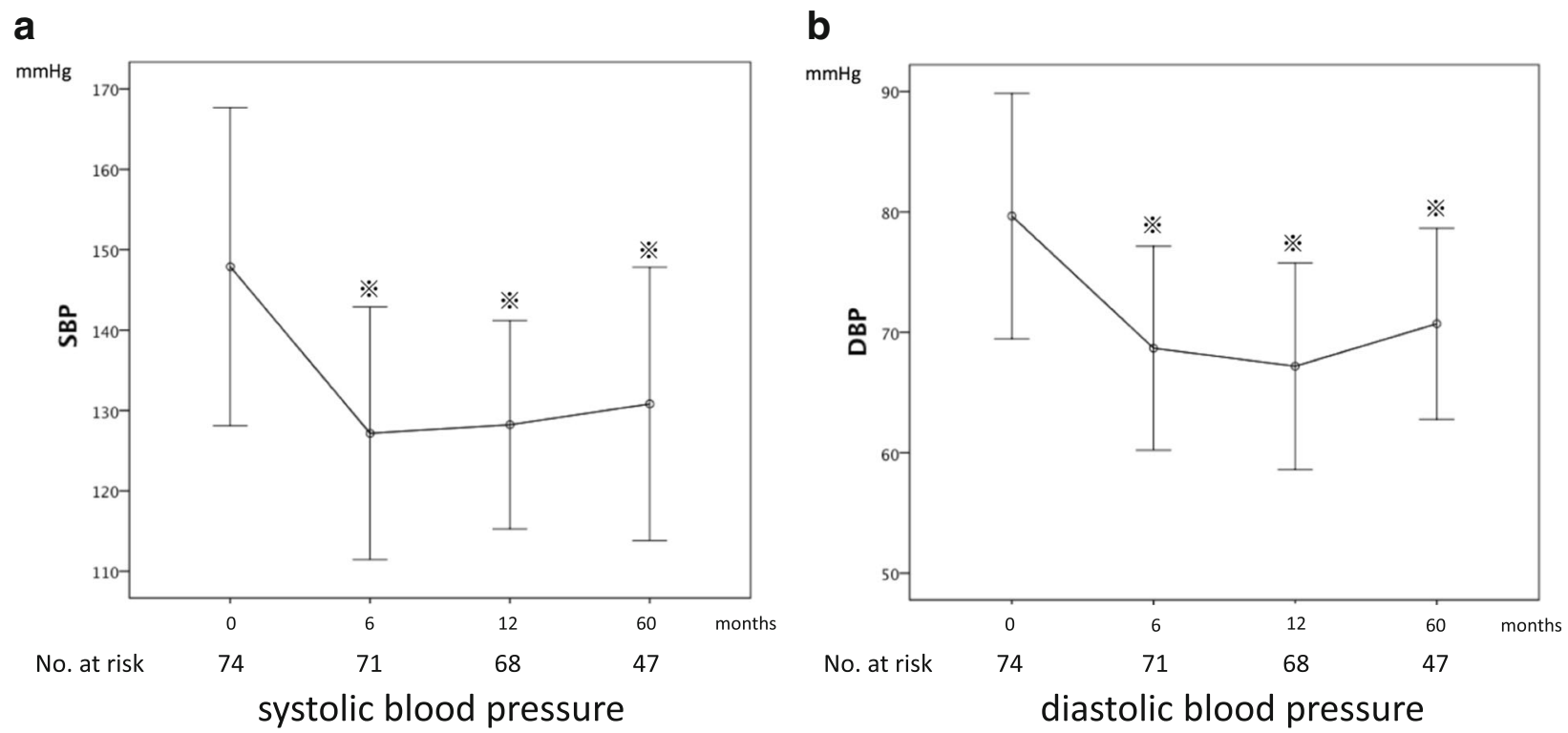

Fig. 4 Changes of systolic and diastolic blood pressure after PTRA. a Systolic blood pressure and $\mathbf{b}$ diastolic blood pressure were significantly lower at 6 months, 1 year, and 5 years after PTRA $(p<0.001) . ※ p<0.001$ vs. the pretreatment value. SBP systolic blood pressure, DBP diastolic blood pressure 
Table 2 Complications and mortality

\begin{tabular}{ll}
\hline Observation period, median(IQR) & $83(49,106)$ months \\
\hline Renal death, $n(\%)$ & $4(5)$ \\
Heart failure, $n(\%)$ & $17(24)$ \\
CCE, $n(\%)$ & $4(5.4)$ \\
Re-PTRA, $n(\%)$ & $10(14)$ \\
Mortality, $n(\%)$ & $14(19)$ \\
Cardiac & $6 / 14(43)$ \\
Cancer & $5 / 14(36)$ \\
Infection & $2 / 14(14)$ \\
Mesenteric infarction & $1 / 14(7)$ \\
\hline
\end{tabular}

CCE cholesterol crystal embolism

PTRA percutaneous transluminal renal angioplasty

patient) after initial PTRA. In addition, cutaneous CCE occurred in 1 patient after second PTRA. Three patients out of four with CCE had 90\% renal stenosis, and one patient had 99\% renal stenosis. Although they were treated with steroid therapy, two out of four patients had renal death and initiated hemodialysis. The one of two had renal death at 4 months $(\mathrm{S}-\mathrm{Cr} ; 2.14 \mathrm{mg} / \mathrm{dl} \Rightarrow$ $7.4 \mathrm{mg} / \mathrm{dl})$, and the other had renal death at 1 month (S$\mathrm{Cr} ; 1.65 \mathrm{mg} / \mathrm{dl} \Rightarrow 7.97 \mathrm{mg} / \mathrm{dl}$ ) after PTRA, respectively. One patient among resting two patients with CCE died of mesenteric artery necrosis at 3 months after PTRA. Fourth patient did not have renal death at 108 months $(\mathrm{S}-\mathrm{Cr} ; 1.06 \mathrm{mg} / \mathrm{dl} \Rightarrow 1.73 \mathrm{mg} / \mathrm{dl})$.

\section{Re-stenosis}

All patients who underwent re-renal angiography showed significant restenosis and underwent re-PTRA. As the results, 10 patients (14\%) underwent re-PTRA. Two patients had experienced PTRA more than twice. The period from first PTRA to re-PTRA was 31 months in median.

\section{Discussion}

Our interventional cohort study demonstrated that PTRA had decreased blood pressure and preserved renal function in patients with severe ARAS for long-term. Thus, PTRA might be beneficial for both the renal and vital prognosis of severe ARAS patients if we carefully consider the indications. We should be clear about the purpose of PTRA in ARAS patients (i.e., for renoprotective, cardio-protective, and/or improved survival).

The ASTRAL [5] and CORAL [6] studies included many patients with mild ARAS ( $\leq 75 \%$ stenosis) among the subjects undergoing PTRA. In such patients' groups, both studies showed that PTRA was not superior to medical therapy with regard to preserving renal function and improving patient survival. However, we thought that these studies might not truly reflect the long-term effectiveness of PTRA for more severe ARAS patients.
Therefore, we focused on ARAS patients with $\geq 75 \%$ stenosis $(84 \pm 8.7 \%)$ and followed them for the longer period (median 83 months) than the previous RCTs (median follow-up period in ASTRAL and CORAL was 33.6 and 43 months).

Regarding the natural course of ARAS, it has been reported as progressive disease. In ARAS patients with > $60 \%$ stenosis, the major renal axis will decrease by $>$ $1 \mathrm{~cm}$ after 1 year in $20 \%$ of total patients [8]. It has also been reported that $40 \%$ of renal arteries $>75 \%$ stenosis undergo occlusion within 12 months [9]. Even if the treatment is given with an angiotensin type 1 receptor blocker (ARB) or angiotensin-converting enzyme inhibitor (ACEi), ARAS itself has been reported to be progressive [10] and lead to renal atrophy [11] and end-stage renal disease (ESRD). Van et al. reported that the prevalence of ARAS at initiating hemodialysis is not infrequent, and ARAS is an important cause of ESRD [12]. However, in our study, the serum creatinine levels and renal size were preserved for long-term after PTRA. Therefore, we thought PTRA might protect the progression of ischemic kidney and decrease renal death in severe ARAS patients.

We should not only focus on the severity of ARAS but also the viability of the kidney itself. The median length of the major axis of treated kidneys was $94 \mathrm{~mm}$ in our study, and renal atrophy (major axis $<85 \mathrm{~mm}$ ) was only noted in $17 \%$ (16 out of 92 kidneys). Serum creatinine levels in patients with renal major axis $<85 \mathrm{~mm}$ at PTRA significantly increased after 60 months post PTRA. However, the levels of serum creatinine in patients with renal major axis $85 \mathrm{~mm}$ or more at PTRA did not change after PTRA up to 84 months. From the viewpoint of renal protection, severe ARAS with sufficient residual kidney function and length seems to be the most appropriate indication for PTRA. Bilateral severe ARAS might be another appropriate indication for actively performing PTRA. Initiation of ACEi or ARB therapy in such patients could decrease systemic blood pressure and exacerbate ischemia of both kidneys which might even trigger the onset of flush pulmonary edema (cardio-renal syndrome type 3) [13]. It seems to be very important to detect ARAS before irreversible severe kidney damage occurs due to renal ischemia.

PSV and RI might be expected to increase along with the severity of RAS. However, our data showed no difference in RI among low, intermediate, and high-grade groups. PSV and EDV in high-grade group were significantly lower than those in other groups. It might be the reason why RI did not differ between groups in our study. Blood flow accelerates by passing through stenotic lumen. However, our result indicated that blood flow decreases in cases of high-grade group or nearly occluding RAS. Therefore, we might be careful not to use sole 
Doppler echography as final diagnostic tool for RAS. Sole Doppler echography to evaluate PSV, EDV, and RI has a risk for missing true very severe RAS. Doppler echography is a functional test that evaluates accelerating blood flow. When we suspect RAS, an imaging test other than Doppler echography to prove stenosis morphologically might be necessary.

PTRA could control resistive hypertension with preserving renal function. Both the systolic and diastolic blood pressure in our study were obviously lower, and a number of anti-hypertension drugs decreased from baseline after PTRA. Among our subjects, $64 \%$ of patients with unilateral stenosis and $72 \%$ of patients with bilateral stenosis had been ingested ARB or ACEi before PTRA. However, blood pressure further decreased even in these patients after PTRA and serum creatinine levels decreased at 1 and 2 years after PTRA in $>95 \%$ stenotic group. In this group, renal ischemia might be further promoted by taking RAS blocker, therefore both renal ischemia and renal function might be improved by PTRA. Previous randomized studies $[5,6]$ have demonstrated a decrease of blood pressure by additional medications. However, renal function did not worsen. In the randomized study, blood pressure might not be fully controlled by first medications. As a result, RAS blocker might not have caused further renal ischemia.

Regarding the cardiac prognosis, the prevalence of congestive heart failure in this study was very high (24\%) at 129 months compared with the ASTRAL and CORAL studies. This may be due to very high baseline frequency of ischemic heart disease (92\%). Besides the cardiac prognosis, we evaluated LV mass at baseline and 12 months after PTRA. We found regression of LV mass in $60 \%$ of the patients, although it was not statistically significant. Improved renal blood flow after PTRA might have decreased stimulation of the renin-angiotensin axis, and thus might have ameliorated cardiac hypertrophy. In fact, the admission rate for congestive heart failure was very low in PTRA group and recurrent heart failure was decreased compared with the medical groups in previous studies [14-16].

The all-cause mortality rate was $19 \%$ at 83 months after PTRA, and the overall survival rate was $85 \%$ at 60 months. It is well known that deterioration of renal function results in decreased phosphate excretion. Hyperphosphatemia is an important arteriosclerotic risk factor for cardiac events and cardiac death [17-19]. Furthermore, ischemic and hypoxic kidney was recently reported to associate with systemic inflammations (neutrophil gelatinase-associated lipocalin (NGAL) and monocyte chemoattractant protein-1 (MCP-1)) [20]. Herrmann et al. showed that cortical hypoxia improve after PTRA. Wan et al. documented that the serum NGAL and MCP-1 decrease after PTRA [21]. In light of these points, PTRA might indirectly decrease mortality via decreasing the risk of worsening renal function, i.e., hyperphosphatemia, cortical hypoxia, and systemic inflammations, by improving direct renal blood flow.

There were several limitations in this study. First, it was a single-center retrospective cohort study that included a relatively small number of patients. Second, this study did not have a control group with $\geq 75 \%$ stenosis who did not undergo PTRA. Because the patient characteristics were different, it was not able to compare our results with those of ASTRAL and CORAL study. Further studies are necessary to confirm the true long-term effectiveness of PTRA in patients with severe ARAS.

\section{Conclusion}

We demonstrated that PTRA significantly decreased blood pressure and preserved renal function for long period in patients with severe ARAS. PTRA might be a useful treatment option for the patients with severe RAS, when we strictly consider the indication of PTRA.

\section{Additional files}

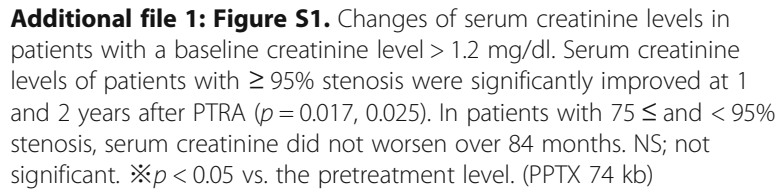

Additional file 1: Figure S1. Changes of serum creatinine levels in patients with a baseline creatinine level $>1.2 \mathrm{mg} / \mathrm{dl}$. Serum creatinine levels of patients with $\geq 95 \%$ stenosis were significantly improved at 1 and 2 years after PTRA $(p=0.017,0.025)$. In patients with $75 \leq$ and $<95 \%$ stenosis, serum creatinine did not worsen over 84 months. NS; not significant. $※ p<0.05$ vs. the pretreatment level. (PPTX $74 \mathrm{~kb}$ )

Additional file 2: Figure S2. Changes of renal major axis (renal size) $※ p<0.01$ vs. the pretreatment value. (PPTX $60 \mathrm{~kb}$ )

\section{Abbreviations}

ACEi: Angiotensin-converting enzyme inhibitor; ANOVA: Analysis of variance; ARAS: Atherosclerotic renal artery stenosis; ARB: Angiotensin type 1 receptor blocker; ASTRAL: The Angioplasty and Stenting for Renal Artery Lesions; CCE: Cholesterol crystal embolism; CORAL: The Cardiovascular Outcomes in Renal Atherosclerotic Lesions; CT: Computed tomography; eGFR: Estimated glomerular filtration rate; ESRD: End-stage renal disease; IRB: Institutional Review Board; J-RAS: Japan multi-central Renal Artery Stent study; LV: Left ventricular; MRA: Magnetic resonance angiography; PSV: Peak systolic velocity; PTRA: Percutaneous transluminal renal angioplasty; RAR: Renal-aortic ratio; RAS: Renal artery stenosis; $\mathrm{RCT}$ : Randomized controlled trial; RI: Resistive index; RRT: Renal replacement therapy; SD: Standard deviation

\section{Acknowledgements}

The author is grateful to our clinical research teams.

\section{Funding}

There was no funding.

Availability of data and materials

The datasets analyzed during the present study are available from the corresponding author on reasonable request.

\section{Authors' contributions}

Mochida $Y$ was involved in the study design, study procedure implementation, data collection, and article writing of the manuscript. All other authors contributed to the study design, data analysis, and article writing of the manuscript. They read and approved the final manuscript. 


\section{Ethics approval and consent to participate}

The study was approved by the Ethical Committee of Tokushukai Group Institutional Review Board (approval number IRB no. TGE00532-024) and was conducted under the Declaration of Helsinki. This report adopted an opt-out consent instead of the written informed consent. We provided patients with information explaining the proposed research project (the purpose, required individual data, and duration of research) by means of an information sheet or a website of the hospital, and gave them the opportunity of opt-out.

\section{Consent for publication}

Not applicable.

\section{Competing interests}

The authors declare that they have no competing interests.

\section{Publisher's Note}

Springer Nature remains neutral with regard to jurisdictional claims in published maps and institutional affiliations.

\section{Author details}

'Kidney Disease and Transplant center, Shonan Kamakura General Hospital, 1370-1 Okamoto, Kamakura, Kanagawa 247-8533, Japan. ${ }^{2}$ Department of Cardiology, Nagano Red Cross Hospital, Nagano, Japan. ${ }^{3} J a p a n$ and Department of Cardiology, Shonan Kamakura General Hospital, Kamakura, Kanagawa, Japan.

Received: 1 June 2017 Accepted: 12 January 2018

Published online: 14 February 2018

\section{References}

1. Zuccala A, Losinno F, Gaggi R, Zucchelli P. Late improvement of renal function in patients treated by percutaneous transluminal renal angioplasty. Contrib Nephrol. 1996;119:74-7.

2. Airoldi F, Palatresi S, Marana I, Bencini C, Benti R, Lovaria A, Alberti C, Nador B, Nicolini A, Longari V, Gerundini P, Morganti A. Angioplasty of atherosclerotic and fibromuscular renal artery stenosis: time course and predicting factors of the effects on renal function. Am J Hypertens. 2000;13:1210-7.

3. Watson PS, Hadjipetrou P, Cox SV, Piemonte TC, Eisenhauer AC. Effect of renal artery stenting on renal function and size in patients with atherosclerotic renovascular disease. Circulation. 2000;102:1671-7.

4. Morganti A, Bencini C, Del Vecchio C, Strata M. Treatment of atherosclerotic renal artery stenosis. J Am Soc Nephrol. 2002;13(Suppl 3):S187-9.

5. Investigators A, Wheatley K, Ives N, Gray R, Kalra PA, Moss JG, Baigent C, Carr S, Chalmers N, Eadington D, Hamilton G, Lipkin G, Nicholson A, Scoble J. Revascularization versus medical therapy for renal-artery stenosis. N Engl J Med. 2009;361:1953-62.

6. Cooper CJ, Murphy TP, Cutlip DE, Jamerson K, Henrich W, Reid DM, Cohen DJ, Matsumoto AH, Steffes M, Jaff MR, Prince MR, Lewis EF, Tuttle KR, Shapiro JI, Rundback JH, Massaro JM, RB D’A Sr, Dworkin LD, Investigators C. Stenting and medical therapy for atherosclerotic renal-artery stenosis. $\mathrm{N}$ Engl J Med. 2014;370:13-22.

7. Fujihara M, Yokoi Y, Abe T, Soga Y, Yamashita T, Miyashita Y, Nakamura M, Yokoi H, Ito S, Investigators JRS. Clinical outcome of renal artery stenting for hypertension and chronic kidney disease up to 12 months in the J-RAS study prospective, single-arm, multicenter clinical study. Circ J. 2015;79:351-9.

8. Guzman RP, Zierler RE, Isaacson JA, Bergelin RO, Strandness DE Jr. Renal atrophy and arterial stenosis. A prospective study with duplex ultrasound. Hypertension. 1994;23:346-50.

9. Schreiber MJ, Pohl MA, Novick AC. The natural history of atherosclerotic and fibrous renal artery disease. Urol Clin North Am. 1984;11:383-92.

10. Zierler RE, Bergelin RO, Davidson RC, Cantwell-Gab K, Polissar NL, Strandness $\mathrm{DE}$ Jr. A prospective study of disease progression in patients with atherosclerotic renal artery stenosis. Am J Hypertens. 1996;9:1055-61.

11. Caps MT, Zierler RE, Polissar NL, Bergelin RO, Beach KW, Cantwell-Gab K, Casadei A, Davidson RC, Strandness DE Jr. Risk of atrophy in kidneys with atherosclerotic renal artery stenosis. Kidney Int. 1998;53:735-42.

12. van Ampting JM, Penne EL, Beek FJ, Koomans HA, Boer WH, Beutler JJ. Prevalence of atherosclerotic renal artery stenosis in patients starting dialysis. Nephrol Dial Transplant. 2003;18:1147-51.

13. Ronco C, Haapio M, House AA, Anavekar N, Bellomo R. Cardiorenal syndrome. J Am Coll Cardiol. 2008;52:1527-39.
14. Kane GC, Xu N, Mistrik E, Roubicek T, Stanson AW, Garovic VD. Renal artery revascularization improves heart failure control in patients with atherosclerotic renal artery stenosis. Nephrol Dial Transplant. 2010;25:813-20.

15. Bloch MJ, Trost DW, Pickering TG, Sos TA, August P. Prevention of recurrent pulmonary edema in patients with bilateral renovascular disease through renal artery stent placement. Am J Hypertens. 1999;12:1-7.

16. Gray BH, Olin JW, Childs MB, Sullivan TM, Bacharach JM. Clinical benefit of renal artery angioplasty with stenting for the control of recurrent and refractory congestive heart failure. Vasc Med. 2002;7:275-9.

17. Dhingra R, Sullivan LM, Fox CS, Wang TJ, RB D'A Sr, Gaziano JM, Vasan RS. Relations of serum phosphorus and calcium levels to the incidence of cardiovascular disease in the community. Arch Intern Med. 2007;167:879-85.

18. Menon V, Greene T, Pereira AA, Wang X, Beck GJ, Kusek JW, Collins A Levey AS, Sarnak MJ. Relationship of phosphorus and calcium-phosphorus product with mortality in CKD. Am J Kidney Dis. 2005:46:455-63.

19. Tonelli M, Sacks F, Pfeffer M, Gao Z, Curhan G. Relation between serum phosphate level and cardiovascular event rate in people with coronary disease. Circulation. 2005;112:2627-33.

20. Hermann SM, Saad A, Eirin A, Woollard J, Tang H, MA MK, Misra S, Glockner JF, Lerman LO, Textor SC. Differences in GFR and tissue oxygenation, and interactions between Stenotic and Contralateral kidneys in unilateral atherosclerotic Renovascular disease. Clin J Am Soc Nephrol. 2016;1 1:458-69.

21. Wang W, Saad A, Herrmann SM, Eirin Massat A, MA MK, Misra S, Lerman LO, Textor SC. Changes in inflammatory biomarkers after renal revascularization in atherosclerotic renal artery stenosis. Nephrol Dial Transplant. 2016;

\section{Submit your next manuscript to BioMed Central and we will help you at every step:}

- We accept pre-submission inquiries

- Our selector tool helps you to find the most relevant journal

- We provide round the clock customer support

- Convenient online submission

- Thorough peer review

- Inclusion in PubMed and all major indexing services

- Maximum visibility for your research

Submit your manuscript at www.biomedcentral.com/submit
Biomed Central 